\title{
A Fermented Milk Product Containing B. lactis CNCM I-2494 Improves the Tolerance of a Plant-Based Diet in Patients with Disorders of Gut-Brain Interactions
}

\author{
Boris Le Nevé $^{1, *(D)}$, Adrian Martinez-De la Torre ${ }^{2,3,4}$ (D) Julien Tap ${ }^{1}$ (D) Adoración Nieto Ruiz ${ }^{2,3,4}$, \\ Muriel Derrien ${ }^{1}{ }^{1}$, Aurélie Cotillard ${ }^{1}$, Jean-Michel Faurie ${ }^{1}$, Elizabeth Barba 2,3,4,5, Marianela Mego 2,3,4, \\ Quentin Dornic 1, John Butler 6, Xavi Merino 2,3,4, Beatriz Lobo 2,3,4, Ferran Pinsach Batet 2,3,4, Marta Pozuelo 2,3,4, \\ Javier Santos $2,3,4$, Francisco Guarner ${ }^{2,3,4}$, Chaysavanh Manichanh $2,3,4$ (D) and Fernando Azpiroz $2,3,4, *$ (D) \\ 1 Danone Nutricia Research, 91767 Palaiseau, France; Julien.TAP@danone.com (J.T.); \\ Muriel.DERRIEN@danone.com (M.D.); aurelie.cotillard@danone.com (A.C.); \\ Jean-Michel.FAURIE@danone.com (J.-M.F.); quentin.dornic@orange.fr (Q.D.) \\ 2 Digestive System Research Unit, University Hospital Vall d'Hebron, 08035 Barcelona, Spain; \\ adriadelatorre@hotmail.com (A.M.-D.l.T.); anieto@vhebron.net (A.N.R.); ebarbaorozco@gmail.com (E.B.); \\ marianelamego@hotmail.com (M.M.); xavier.merino@uab.cat (X.M.); beatriz.lobo@vhir.org (B.L.); \\ f.pinsach.batet@gmail.com (F.P.B.); mpozud00@gmail.com (M.P.); javier.santos@vhir.org (J.S.); \\ fguarner@icloud.com (F.G.); cmanicha@gmail.com (C.M.) \\ 3 Centro de Investigación Biomédica en Red de Enfermedades Hepáticas y Digestivas (Ciberehd), \\ Departament de Medicina, Universitat Autònoma de Barcelona, 08193 Cerdanyola del Vallés, Spain \\ check for \\ updates \\ Citation: Nevé, B.L.; Martinez-De la \\ Torre, A.; Tap, J.; Ruiz, A.N.; Derrien, \\ 4 Departament de Medicina, Universitat Autònoma de Barcelona, 08193 Cerdanyola del Vallès, Spain \\ 5 Department of Gastroenterology, Hospital Clínic, 08036 Barcelona, Spain \\ 6 Lawson Health Research Institute, London ON N6C 2R5, Canada; jbutler@lawsonimaging.ca \\ * Correspondence: boris.le-neve@danone.com (B.L.N.); azpiroz.fernando@gmail.com (F.A.)
} M.; Cotillard, A.; Faurie, J.-M.; Barba, E.; Mego, M.; Dornic, Q.; et al. A Fermented Milk Product Containing B. lactis CNCM I-2494 Improves the Tolerance of a Plant-Based Diet in Patients with Disorders of Gut-Brain Interactions. Nutrients 2021, 13, 4542. https://doi.org/10.3390/nu13124542

Academic Editor: Stefano Guandalini

Received: 12 November 2021 Accepted: 12 December 2021 Published: 18 December 2021

Publisher's Note: MDPI stays neutral with regard to jurisdictional claims in published maps and institutional affiliations.

Copyright: (c) 2021 by the authors. Licensee MDPI, Basel, Switzerland. This article is an open access article distributed under the terms and conditions of the Creative Commons Attribution (CC BY) license (https:// creativecommons.org/licenses/by/ $4.0 /)$.

\begin{abstract}
Healthy, plant-based diets, rich in fermentable residues, may induce gas-related symptoms. The aim of this exploratory study was to assess the effects of a fermented milk product, containing probiotics, on the tolerance of a healthy diet in patients with disorders of gut-brain interactions (DGBI), complaining of excessive flatulence. In an open design, a 3-day healthy, mostly plant-based diet was administered to patients with DGBI (52 included, 43 completed) before and at the end of 28 days of consumption of a fermented milk product (FMP) containing Bifidobacterium animalis subsp. lactis CNCM I-2494 and lactic acid bacteria. As compared to a habitual diet, the flatulogenic diet increased the perception of digestive symptoms (flatulence score $7.1 \pm 1.6$ vs. $5.8 \pm 1.9 ; p<0.05$ ) and the daily number of anal gas evacuations $(22.4 \pm 12.5$ vs. $16.5 \pm 10.2 ; p<0.0001)$. FMP consumption reduced the flatulence sensation score (by $-1.6 \pm 2.2 ; p<0.05$ ) and the daily number of anal gas evacuations (by $-5.3 \pm 8.2 ; p<0.0001$ ). FMP consumption did not significantly alter the overall gut microbiota composition, but some changes in the microbiota correlated with the observed clinical improvement. The consumption of a product containing B. lactis CNCM I-2494 improved the tolerance of a healthy diet in patients with DGBI, and this effect may be mediated, in part, by the metabolic activity of the microbiota.
\end{abstract}

Keywords: flatulence; fermentable carbohydrates; probiotics; microbiota; digestive symptoms; disorders of gut-brain interactions; B. lactis DN-173010

\section{Introduction}

A healthy diet requires a proper balance of nutrients, vitamins and minerals for one's own metabolism, but also many residues that serve as substrates for the intestinal microbiota, the largest pool of symbiotic microorganisms in the human body. The metabolic processing of diet residues by the microbiota involves fermentative pathways that release gas, and in susceptible individuals, this may be associated with the perception of digestive symptoms. 
A large proportion of patients in clinical practice complain of digestive symptoms, such as bloating, abdominal distension, and flatulence, in the absence of structural abnormalities, and attribute their complaints to intestinal gas. These patients exhibit susceptibility of the alimentary tract, with poor tolerance of residue-rich healthy diets. This may have further implications because some data indicate that these patients have enteric dysbiosis, and a diet poor in residues may further deteriorate their microbiota.

Previous studies indicate that the administration of specific living microorganisms (probiotics) may improve gas-related symptoms. Indeed, it has been shown that a fermented milk product containing Bifidobacterium animalis subsp. lactis CNCM I-2494 and lactic acid bacteria improves symptoms and well-being in women with mild digestive complaints [1-4], and reduces bloating, digestive discomfort, and abdominal distension in IBS-C patients [5,6]. Furthermore, the mechanism of action of this probiotic seems to be related to the metabolism of the microbiota [7].

The aim of the present study was to determine the efficacy of a fermented milk product (FMP) containing B. lactis CNCM I-2494 and lactic acid bacteria on the tolerance of a healthy diet in susceptible individuals. To this aim, we recruited a pool of patients with moderate to severe symptoms in response to a healthy diet containing an adequate load of plant residues, and evaluated the effect of the FMP on subjective clinical parameters (gas-related symptoms) and objective physiological parameters related to intestinal gas metabolism.

\section{Materials and Methods}

\subsection{Study Design and General Procedure}

Single-centre, open-label study performed in a tertiary care referral centre on the effect of a fermented milk product (FMP) on intestinal gas production and gastrointestinal symptoms. The study consisted of an 18-day run-in phase and a 28-day FMP administration phase (Figure 1). The main outcomes were gastrointestinal symptoms, number of anal gas evacuations, and volume of anal gas evacuated following a probe meal measured at the end of the run-in phase and the administration phase.

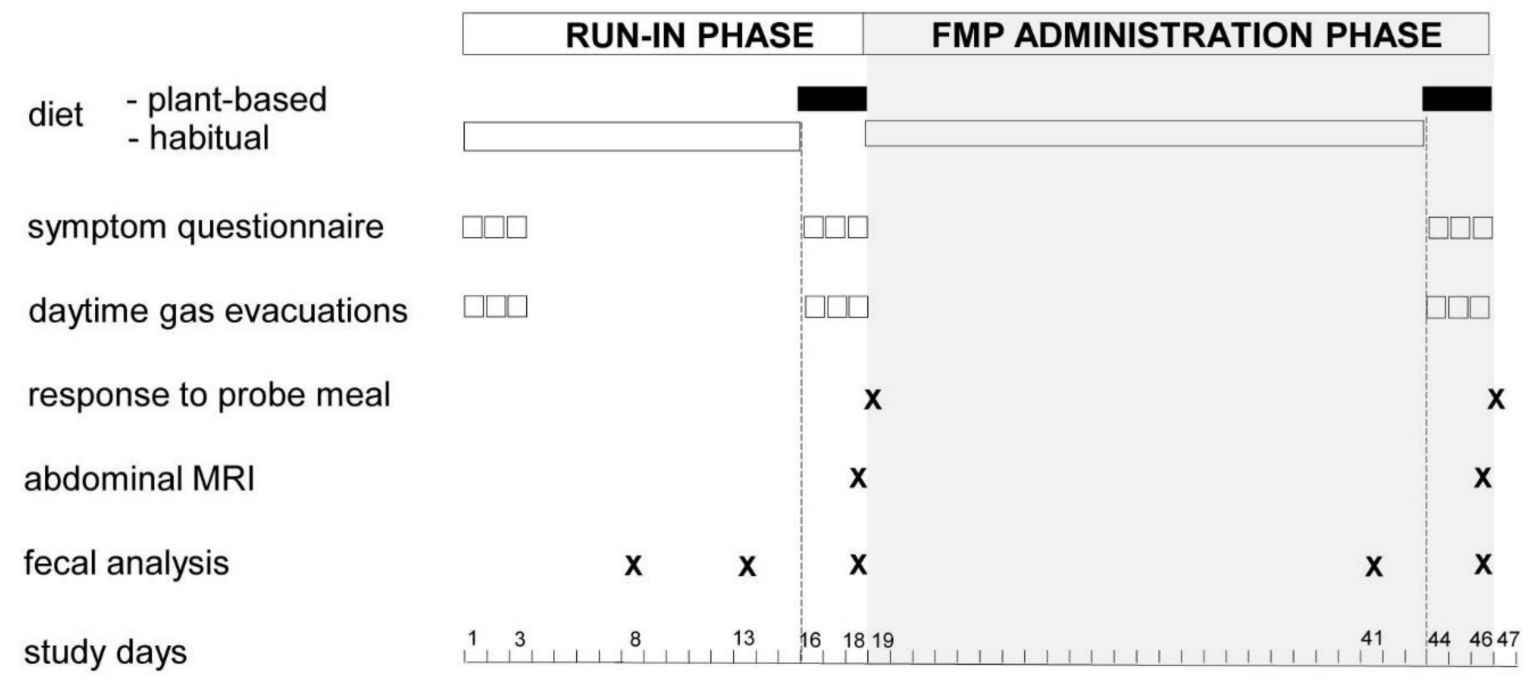

Figure 1. Study design. FMP: fermented milk product. Abdominal MRI scheduled in half of the participants.

During the study, participants were instructed to consume their habitual diet except during the last 3 days of the run-in phase (days 16-18) and the administration phase (days 44-46) when a mostly plant-based diet (see below) was administered. Participants were not allowed to consume any fermented dairy products (with or without probiotics) or any tablets, pills, or food supplements containing pre- or probiotics other than those provided during the study. The food items of the plant-based diet and the fermented milk product were provided by the investigators. 
The clinical study was conducted according to the Declaration of Helsinki. The study protocol had previously been approved by the Institutional Review Board of the University Hospital Vall d'Hebron (Comitè d'Ėtica d'Investigació Clinica, Vall d'Hebron Insititut de Recerca; protocol number PR-AG 292/2012, approved 29 January 2016) and all participants provided written informed consent. The protocol was also registered with ClinicalTrials.gov (NCT02936713) and included separate pilot studies in healthy subjects [8] and in patients with disorders of gut-brain interactions (reported here). All authors had access to the study data and reviewed and approved the final manuscript.

\subsection{Participants}

Patients with DGBI (both genders; 18-75 year age range; body mass index (BMI) $18.5-30 \mathrm{~kg} / \mathrm{m}^{2}$ ) diagnosed by Rome III criteria participated in the study using previously validated clinical questionnaires [9-12]. Patients were recruited from the outpatient clinics, both on campus and in the community, serviced by the Vall d'Hebron Digestive Diseases Department. Before inclusion, patients were required to have flatulence ( $\geq 5$ on a $0-10$ scale), abdominal bloating or discomfort ( $\geq 3$ on a $0-10$ scale) and negative sensation of digestive well-being during the previous week. The day after the run-in phase (day 19) the symptom questionnaires related to days 16-18 (on the plant-based diet) were reviewed, and the following continuation criteria were required to enter the administration phase: (a) prospective confirmation of entry criteria (described above), (b) $\geq 8$ anal gas evacuations per day, and (c) $\geq 50 \%$ daily compliance to the plant-based diet (calculated as the percent intake per day of the total fibre content in the diet). Intake of antibiotics during the previous two months, changes in dietary habits in the previous 4 weeks, antecedents of digestive surgery (except for appendectomy and cholecystectomy performed more than two years before), and treatments that might affect the central nervous system or gastrointestinal function (anxiolytics/antidepressants and laxatives at stable dose were allowed) were considered as exclusion criteria.

\subsection{Plant-Based Diet}

The plant-based diet consisted of the following: (a) breakfast of wholemeal cookies $(39 \mathrm{~g})$ plus coffee, tea and/or milk; (b) lunch of white beans (200 g), lentils (200 g) or chickpeas (200 g) plus meat, fowl, fish or eggs, and fruit (banana, figs, peaches, or prunes); (c) dinner of vegetable cream $(200 \mathrm{~mL})$ plus meat, fowl or fish, and fruit (apple or pear). This diet provides $58 \%$ caloric content as carbohydrates, $29 \%$ as proteins and $13 \%$ as fat with $19 \mathrm{~g}$ of fibre per day. Caloric content of the diet was not standardized. Participants were instructed to self-report the foods they consumed during the 3 days on the plant-based diet to assess compliance.

\subsection{Study Product}

During the 28-day administration phase, participants consumed 1 pot (125 g) of the study product at breakfast and another pot at dinner. The study product was a fermented milk containing three Streptococcus salivarius subsp. thermophilus strains (CNCM I-2773, CNCM I-2130, CNCM I-2272), Lactobacillus delbrueckii subsp. bulgaricus (CNCM I-1519), Bifidobacterium animalis subsp. lactis (CNCM I-2494 previously referenced as DN-173010), and Lactococcus lactis subsp. lactis (CNCM I-1631). The study product was manufactured and supplied by Danone Nutricia Research, Palaiseau, France and contained per $\mathrm{g}$ at least $3.4 \times 10^{7}$ colony forming units (cfu) of B. lactis, $1 \times 10^{6} \mathrm{cfu}$ of L. lactis, and $1 \times 10^{7} \mathrm{cfu}$ of $S$. thermophilus and L. bulgaricus.

\subsection{Main Outcomes}

Gastrointestinal symptoms and the number of anal gas evacuations were measured during 3-day periods at the following 3 time points throughout the study: (a) at the beginning of the run-in phase on the habitual diet (days 1-3); (b) at the end of the run-in phase on the plant-based diet (days 16-18); (c) at the end of the administration phase on the plant-based diet 
(days 44-46) (Figure 1). The volume of anal gas evacuated after a probe meal was measured after 3 days on the plant-based diet at the following 2 time points: (a) the day after the run-in phase (day 19); (b) the day after the administration phase (day 47).

\subsubsection{Daily Symptoms Questionnaire}

During the 3 days of each evaluation period, the following gastrointestinal symptoms were self-assessed by the participants using daily questionnaires (0-10 analogue scoring scales): (a) subjective sensation of flatulence; (b) abdominal bloating; (c) abdominal distension; (d) borborygmi; (e) odoriferous flatus; (f) abdominal discomfort/pain. The questionnaire also recorded the following: (g) digestive well-being scored on a scale graded from +5 (extremely pleasant sensation/satisfaction) to -5 (extremely unpleasant sensation/dissatisfaction); (h) frequency of bowel movements; (i) stool consistency using the Bristol stool form scale. The sensitivity and quality of this patient-related outcome have been validated by previous research aiming to assess the effects of dietary interventions on both healthy subjects and patients with DGBIs [12].

\subsubsection{Number of Anal Gas Evacuations}

The number of daytime anal gas evacuations was assessed during each evaluation period. Participants were instructed to carry an event marker (Hand Tally Counter No 101, Digi Sport Instruments, Shangqiu, China) and to use it to record each passage of anal gas during the day. Previous research showed that this method provides reproducible results $[12,13]$ that correlate with the simultaneous recording of anal gas outflow $(R>0.95$; $p<0.05)$ [14].

\subsubsection{Volume of Anal Gas Production after a Probe Meal (Gas Production Test)}

Participants came to the laboratory the morning after an overnight fast to consume a probe meal consisting of whole meal cookies $(96 \mathrm{~g})$ plus coffee and milk ( $54 \mathrm{~g}$ carbohydrates, $7 \mathrm{~g}$ proteins and $21 \mathrm{~g}$ fat with $8 \mathrm{~g}$ fibre; $450 \mathrm{Kcal}$ ). The volume of anal gas evacuated was measured continuously during the $4 \mathrm{~h}$ after the probe meal, as previously described [12,13]. Briefly, anal gas was collected using a rectal balloon catheter (20 F Foley catheter, Bard, Barcelona, Spain) connected via a gas-tight line to a barostat, and the volume was continuously measured. To prevent anal gas leaks, the intrarectal balloon was inflated with $5 \mathrm{~mL}$ of water.

\subsection{Exploratory Outcomes}

\subsubsection{Colonic Gas Content}

Half of the participants were scheduled for colonic gas content measurements. Colonic gas content during consumption of the plant-based diet was measured by abdominal magnetic resonance imaging (MRI) in the run-in phase (day 18) and in the administration phase (day 46), following the procedure described previously [8].

\subsubsection{Faecal Microbiota Analysis}

All participants were given a kit with standard instructions to self-collect their stool samples at home at the following 5 time points throughout the study: (a) during run-in phase on the habitual diet (2 samples on days 8 and 13); (b) during run-in phase on the plantbased diet (day 18); (c) during administration phase on the habitual diet (day 41); (d) during administration phase on the plant-based diet (day 46) (Figure 1). Stool samples were mixed with a spatula provided in the kit to obtain a homogenous mixture, immediately frozen by the participants in their home freezers at $-20^{\circ} \mathrm{C}$ and later brought to the laboratory in a freezer pack, where they were stored at $-80{ }^{\circ} \mathrm{C}$ until further use. Genomic DNA was extracted by mechanical process [15] and faecal microbiota were profiled using $16 \mathrm{~S}$ rRNA gene amplicon sequencing based on Illumina MiSeq technology. Amplicon reads were analysed using QIIME software (1.9.1). Sequences were clustered based on the USEARCH (search and clustering) algorithm (5.2.236v) into operational taxonomic units 
(OTUs), taxonomically assigned using a database combining greengenes (gg_13_8 release) and PATRIC (Pathosystems Resource Integration Center).

\subsection{Statistical Analysis}

Due to the exploratory nature of this study, no sample size calculation or multiplicity adjustments were performed. Analysed populations for the run-in and administration phases were the full analysis set 1 (FAS $1 ; n=47)$ and FAS $2(n=43)$, respectively.

Clinical results are expressed as mean $[95 \% \mathrm{CI}]$. For each parameter, the values of the 2 last days from each evaluation period were averaged for statistical comparisons. Comparisons were performed using Wilcoxon signed-rank test. Statistical tests were two sided with a significance level of $5 \%$ and all confidence intervals are presented as two sided with a confidence level of $95 \%$.

Microbial ecology and statistical analyses were performed using QIIME and R software (3.4.3v). Spearman correlations were used to assess correlations between changes in clinical parameters and faecal microbiota composition, as previously described [8].

\section{Results}

\subsection{Demographics and Compliance to Study Procedures}

Forty-nine subjects with DGBI (39 women, 10 men; $51 \pm 15$ years; $24.9 \pm 3.5 \mathrm{Kg} / \mathrm{m}^{2}$ BMI) were included in the run-in phase (FAS 1); 38 patients fulfilled the criteria of irritable bowel syndrome (20 constipation-predominant, 8 diarrhoea-predominant, 7 mixed type, 3 undetermined), 5 had functional abdominal pain, 3 had functional bloating, and 3 had functional dyspepsia (postprandial distress syndrome). After the run-in phase, 44 patients fulfilled the continuation criteria and entered the administration phase (FAS 2), and 43 completed the study (Figure 2). Adherence to the dietary instructions was high (mean per-protocol compliance $>90 \%$ ) and the study product was well tolerated.

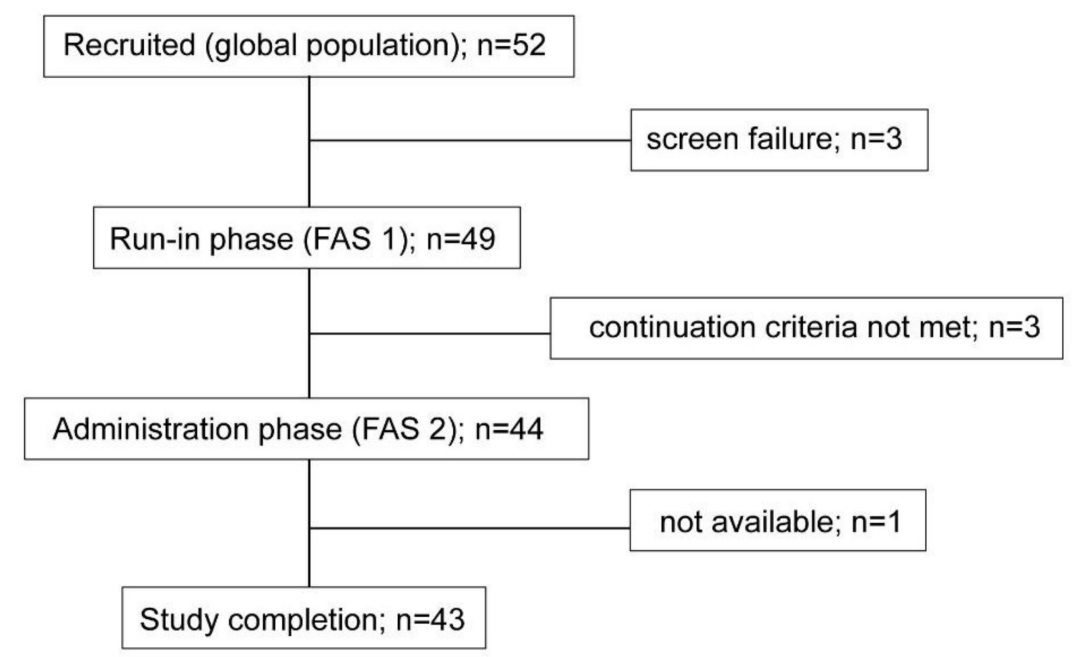

Figure 2. Flow-chart. FAS 1: full analysis set 1; FAS 2: full analysis set 2.

\subsection{Effect of the Plant-Based Diet during the Run-In Phase}

On their habitual diet, patients reported digestive symptoms (flatulence, abdominal discomfort, abdominal distension, bloating, and borborygmi) and odoriferous flatus (Table 1). These symptoms were associated with a negative sensation of digestive wellbeing. On the plant-based diet, digestive symptoms significantly increased with a decrease in the sensation of digestive well-being (Table 1). These subjective changes were associated with a significant increase in the number of daytime anal gas evacuations, without changes in stool frequency and consistency (Table 1). 
Table 1. Effects of the plant-based diet on gas-related symptoms and frequency of anal gas evacuations.

\begin{tabular}{cccc}
\hline & Habitual Diet $(n=48)$ & Plant-Based Diet $(n=47)$ & $p$ Value \\
\hline Flatulence * & $5.8[5.2 ; 6.32]$ & $6.8[6.2 ; 7.4]$ & $p<0.001$ \\
Abdominal & $5.3[4.7 ; 5.9]$ & $6.2[5.7 ; 6.7]$ & $p<0.001$ \\
discomfort/pain * & $5.8[5.1 ; 6.4]$ & $6.7[6.1 ; 7.3]$ & $p<0.001$ \\
Abdominal distension * & $5.7[5.0 ; 6.3]$ & $6.7[6.1 ; 7.3]$ & $p<0.001$ \\
Bloating * & $4.0[3.2 ; 4.8]$ & $4.5[3.8 ; 5.3]$ & $p<0.001$ \\
Borborygmi * & $4.3[3.5 ; 5.1]$ & $4.7[3.9 ; 5.5]$ & $p>0.05$ \\
Odor of flatus * & $-1.8[-2.3 ;-1.2]$ & $-2.5[-2.9 ;-2.1]$ & $p<0.005$ \\
Digestive well-being * & $16.5[13.5 ; 19.4]$ & $22.3[18.6 ; 26.0]$ & $p<0.001$ \\
Anal gas evacuations * & $1.4[1.2 ; 1.6]$ & $1.4[1.2 ; 1.6]$ & $p>0.05$ \\
Bowel movements ** & $4.0[3.6 ; 4.3]$ & $4.0[3.6 ; 4.4]$ & $p>0.05$ \\
Stool consistency ** & & &
\end{tabular}

Data are means [95\% confidence interval] presented on full analysis set 1 (FAS1) population; comparisons by Wilcoxon signed-rank test. Data calculated in each subject using average over last 2 days of each evaluation period $\left.{ }^{*}\right)$ or average over last 3 days of each evaluation period $\left(^{* *}\right)$.

\subsection{Effect of the Fermented Milk Product on the Tolerance of the Plant-Based Diet}

Consumption of the study product improved the tolerance of the healthy plant-based diet, with a significant decrease in digestive symptoms and enhanced sensation of digestive well-being (Figure 3). This effect was associated with a reduction in the number of anal gas evacuations (by $-5.3[-7.8 ;-2.7]$ evacuations per day; $p<0.001$ ).

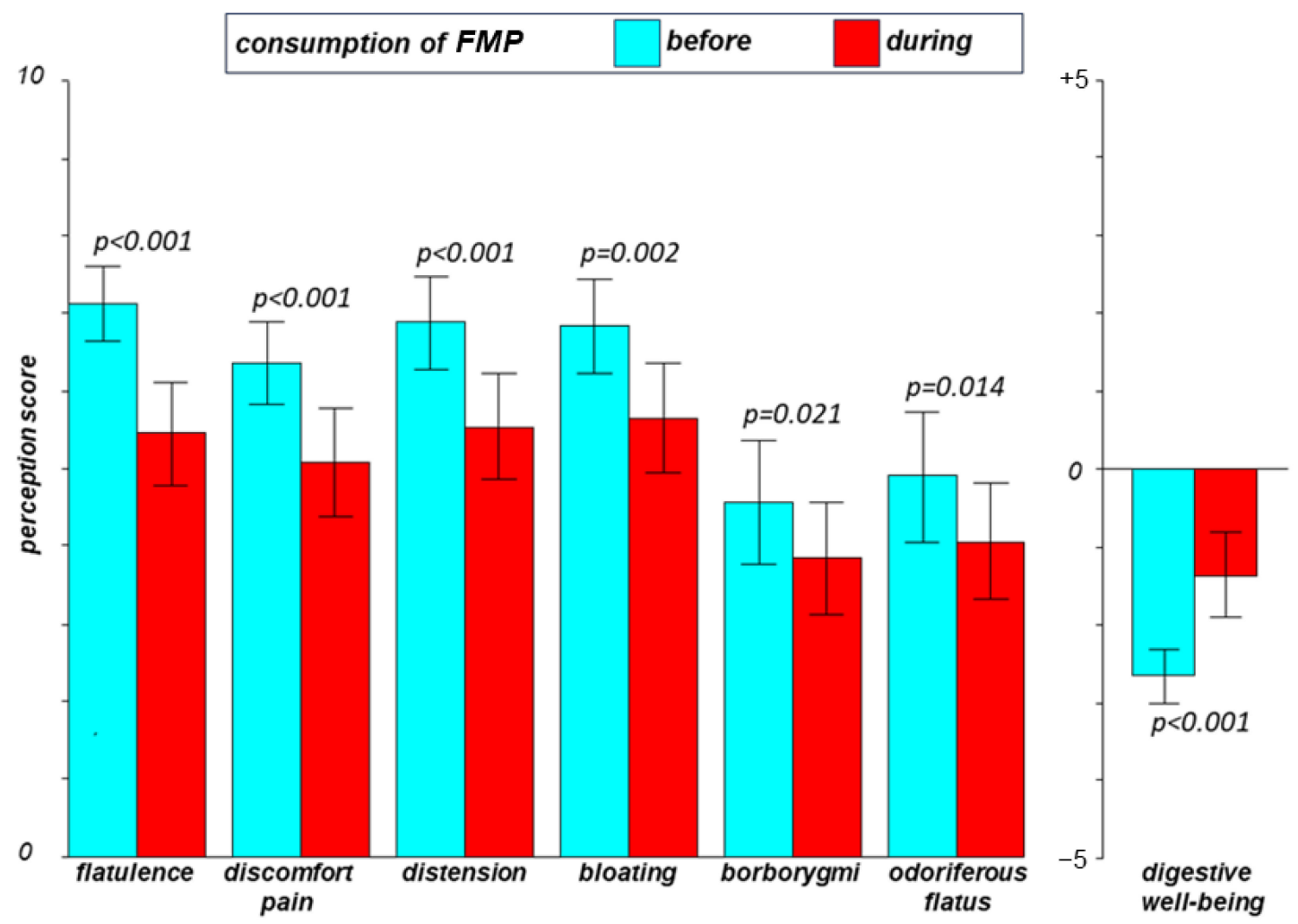

Figure 3. Effect of consumption of fermented milk product (FMP) on tolerance of a plant-based diet. Data are means [95\% confidence interval]. Statistical comparisons were performed by Wilcoxon signed-rank test in each subject using the average of the last 2 days of evaluation periods (on the plant-based diet) before (days 17 and 18) and during FMP consumption (days 45 and $46: n=43$ ).

No effects of FMP consumption were detected on stool frequency and consistency (changes by $-0.08[-0.2 ; 0.0]$ daily bowel movements and 0.17 [ $-0.2 ; 0.5]$ Bristol score), the volume of gas collected during the $4 \mathrm{~h}$ period after ingestion of the probe meal $(139 \mathrm{~mL}$ 
[109; 169] before versus $153 \mathrm{~mL}$ [77; 229] during administration; change by $13 \mathrm{~mL}$ [ $-65 ; 91]$ ), and the volume of colonic gas measured by MRI (total volume of $208 \mathrm{~mL}$ [157; 258] before versus $191 \mathrm{~mL}$ [125; 256] during administration; change by $-16.65 \mathrm{~mL}$ [-77; 43]; paired data from 22 participants).

\subsection{Faecal Microbiota}

A total of 199 faecal samples collected from the participants were used for the microbial community analysis. No differences in the overall gut microbiota composition among the five faecal samples taken during the study were detected by the weighted UniFrac distance. FMP consumption did not elicit significant changes in the faecal microbiota diversity, as shown by the alpha- and beta-diversity (Wilcoxon signed-rank tests, $p>0.05$ for Chao 1 index, $p>0.05$ for weighted UniFrac distance).

Spearman correlation was used to associate the response of the clinical parameters to FMP consumption with changes in faecal microbiota composition. The decrease in the number of anal gas evacuations during FMP consumption correlated with a decrease in the relative abundance of Christensenellaceae ( $r h o=0.56 ; p<0.001$ ) and Bacteroides ( $r$ o $=0.46$; $p<0.006$ ), and an increase in Coriobacteriaceae (rho $=-0.34 ; p=0.042$ ) (Figure $4 \mathrm{~A}$ ). The decrease in flatulence sensation was related to a decrease in the relative abundance of Bacteroides spp. (rho $=0.43 ; p=0.010)$ and Adlercreutzia (rho $=0.39 ; p=0.020)$, and an increase in Peptococcaceae (rho $=-0.43 ; p=0.011)$, Cyanobacteria 4COd 2 Ys2 (rho $=-0.40$; $p=0.018)$, and Methanosphaera (rho $=-0.28 ; p=0.025$ ) (Figure $4 \mathrm{~B})$.
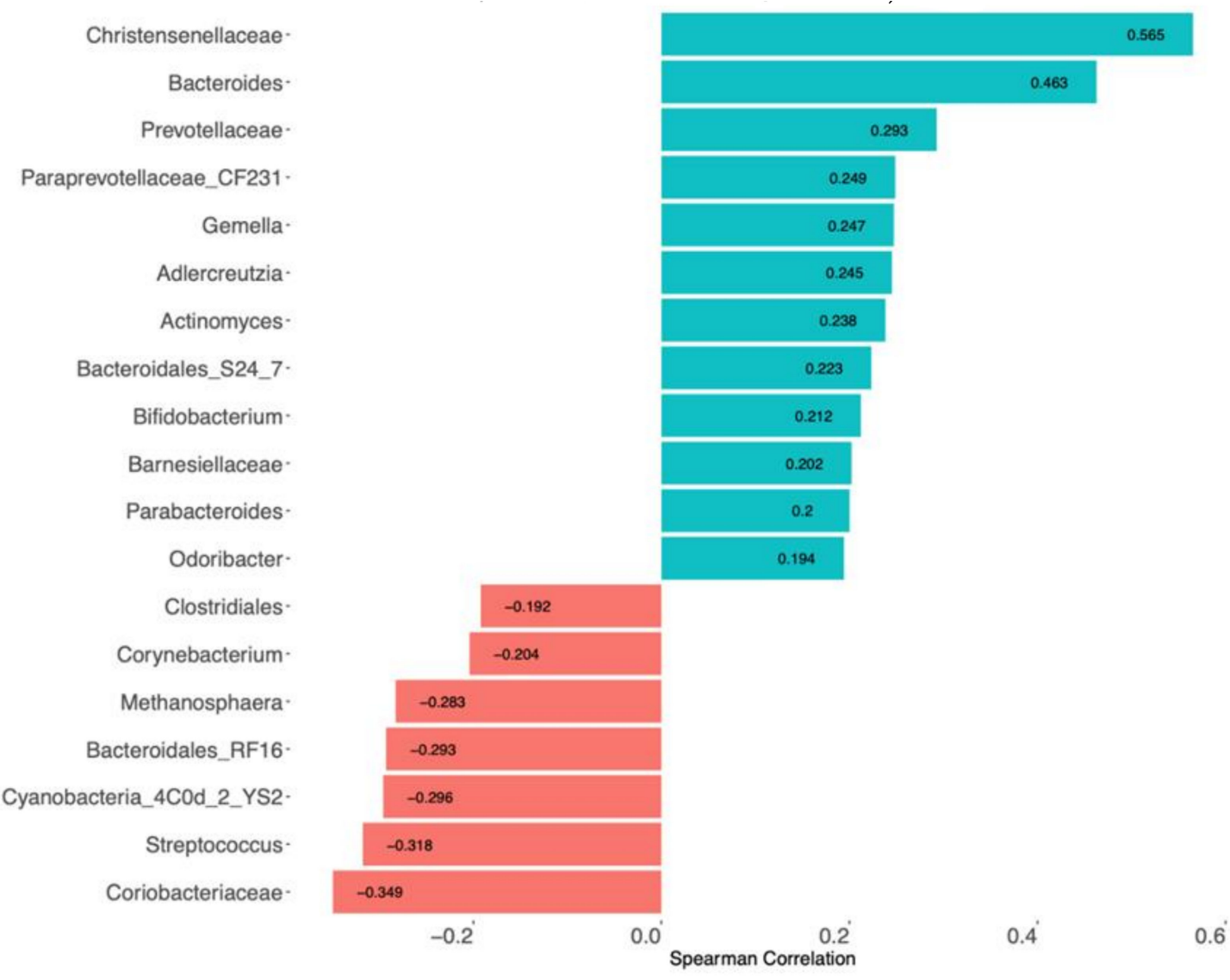

(A)

Figure 4. Cont. 


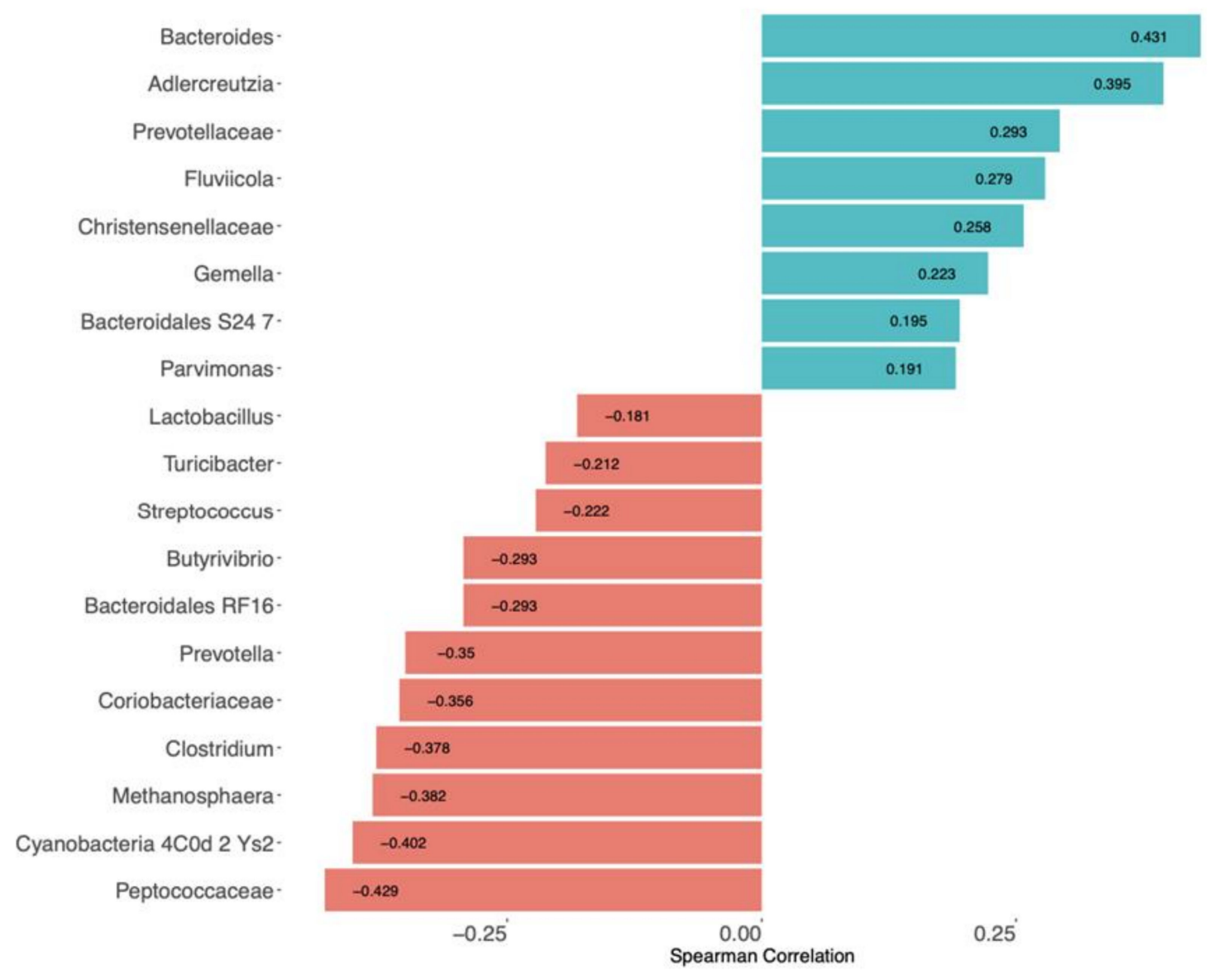

(B)

Figure 4. Spearman correlation between changes in clinical parameters and bacterial genera. (A). Number of anal gas evacuations. (B). Sensation of flatulence. Strength and significance of Spearman correlations (rho coefficient) are indicated in the colour shade. Red: change in microbiota genera negatively associated with the change in clinical parameter. Blue: change in microbiota genera positively associated with the change in clinical parameter. Only the highest correlations are shown.

\section{Discussion}

Our data indicate that the consumption of a fermented milk product (FMP), containing B. lactis CNCM I-2494 and lactic acid bacteria, improves symptoms in patients with disorders of gut-brain interactions (DGBI) on a healthy, plant-based diet. Digestive sensations depend on the balance between the gut content (intraluminal stimuli) and tolerance, i.e., the way the intraluminal content is sensed and managed by the gut. Conceivably, the product improved the tolerance of a healthy plant-based diet acting on these two factors.

When consuming the plant-based diet, patients experienced worsening of their symptoms, and this may be related to the load of fibre, fermentable oligo-, di-, mono-saccharides and polyols (FODMAPs), and other non-absorbable residues. These residues are metabolized by the microbiota, releasing gas, and increase the bulk of colonic biomass, and both mechanisms may be related to the poor tolerance of the diet. Indeed, on the plant-based diet, the number of anal gas evacuations increased, but FMP consumption corrected this effect, indicating that the FMP reduced the amount of gas released from the fermentation of dietary residues. By contrast, in the gas production test, the volume of gas collected during the $4 \mathrm{~h}$ after the probe meal was not influenced by the FMP. This apparent discrepancy may be explained by the overflow of fermentable residues delivered with the probe meal, which might have overcome the potential effect of the product on fermentative activity. The volume of gas within the colon, measured by magnetic resonance, remained constant before and during FMP consumption. This observation, i.e., the effect of the FMP on gas 
metabolism, without changes in the colonic gas content, is in accordance with previous studies that showed no differences in intracolonic gas volume with dietary interventions that modified gas production [16,17]. Indeed, the gas content is under tight homeostatic control at around 100-200 mL. The amount of gas released by fermentation varies greatly depending on the availability of residues. However, the gas production is matched with a very dynamic and effective gas disposal system. The most important mechanism is absorption across the gut-blood barrier and elimination by pulmonary diffusion via breath. About $70 \%$ of the gas produced is rapidly eliminated following this pathway. The hydrogen concentration in the breath was not measured in the present study, but a product with the same B. lactis CNCM I-2494 reduced hydrogen breath excretion after a nutrient meal containing lactulose was given to IBS patients [7]. A fraction of gas is eliminated from the lumen by gas-consuming microorganisms; lactate-producing bacteria present in the FMP may contribute to microbiota gas consumption, but this metabolic process conceivably takes longer, and may not be appreciated shortly after the probe meal.

It has been documented that, as compared to a low-residue diet, a high-residue diet increases both the volume of colonic biomass and faecal clearance, conceivably by an increase in the residue load and microbiota proliferation [16,17]. FMP administration may have moderated this effect, but biomass volumes and faecal weight were not measured in the present study, and we cannot determine whether the effect of the FMP on symptoms was related to the downregulation of biomass proliferation.

In addition to the potential intraluminal effects of the FMP, probiotics may have influenced the way the intestine handled and sensed its content, i.e., intestinal motility and sensitivity. Patients with functional gut disorders exhibit visceral hypersensitivity and reflex dysfunctions; through the interaction of these mechanisms, they develop symptoms in response to stimuli, e.g., diets that are well tolerated by healthy subjects. It has been shown that the administration of a product with the same B. lactis CNCM I-2494 reduced visceral sensitivity to colorectal distension in an experimental rat model [18], and exerted a modulatory effect on the brain regions involved in the central processing of emotion and sensation in healthy women [19]. Furthermore, administration of the same product to patients with IBS-C had a prokinetic effect (accelerated orocecal and colonic transit), associated with an improvement in symptoms [5].

Our 4-week FMP intervention had minor effects on faecal microbiota composition. In a previous study, using shotgun metagenomics, we observed that strong dietary interventions for short periods of time have only minor effects on composition, but a considerable impact on microbiota metabolism, as assessed by changes in the microbial metabolic pathways in faecal samples, and the urinary excretion of microbial metabolites [16]. Our findings in this study may also reflect metabolic adaptation to the plant-based diet during FMP consumption, as shown by the reduction in flatulence scores and anal gas evacuations after FMP consumption, linked with the downregulation of some putative hydrogenproducing taxa (Bacteroides, Christensenellaceae, and Prevotellaceae) and the upregulation of some hydrogen-consuming taxa (Methanospherae). In a previous study, an FMP, containing B. lactis CNCM I-2494, reduced the metabolic potential of the Prevotella/Bacteroides ratio in IBS patients [7]. Here, we could detect a reduction in Prevotellaceae, associated with improved clinical effects, particularly in relation to the number of anal gas evacuations and the sensation of flatulence. A high-fermented-food diet was recently shown to increase faecal microbiota diversity in individuals consuming a median of five fermented food servings per day (including FMPs) for 10 weeks [20]. Our study did not reproduce such findings, most likely due to the shorter duration and lower diversity and/or exposure of the fermented food in our intervention.

\section{Limitations}

We acknowledge that this pilot study was not controlled, and the results must be considered as hypothesis-generating, rather than conclusive. A possible placebo effect on subjective perception cannot be excluded; however, the patients did not know what to 
expect in response to the consumption of the plant-based diet and the product. Several technical limitations, discussed above, had to be compromised due to the complexity of the study and the relatively large sample size. In particular, the microbiota analysis by $16 \mathrm{~S}$ rRNA gene sequencing does not allow for the evaluation of the potential (and plausible) effects of the FMP on microbiota metabolic pathways. Furthermore, we did not evaluate the effect of the product at different times during and after administration, and, hence, we cannot ascertain whether the product may induce sensations by itself that could confound the effects observed on the plant-based diet, in terms of how long it should be administered to be effective and whether the effects are persistent after discontinuation.

\section{Clinical Inferences and Conclusions}

The current management of gas-related symptoms in patients with disorders of gutbrain interactions includes low-residue diets and prebiotics [21-26]. The present study shows that probiotics may offer an alternative approach. Conceivably, the treatment of these patients may benefit from an individualized combination of diet, prebiotics, and probiotics, based on objective indicators related to physiological parameters and microbiota activity.

Author Contributions: Conceptualization, B.L.N. and F.A.; Data curation, Q.D.; Formal analysis, B.L.N., A.M.-D.I.T., J.T., M.D., A.C., Q.D., J.-M.F., J.B., F.P.B., C.M. and F.A.; Funding acquisition, F.A.; Investigation, E.B., M.M., A.N.R., J.B., X.M., B.L., J.S., M.P. and F.A.; Methodology, A.M.-D.1.T., A.C., Q.D. and F.P.B.; Project administration, B.L.N. and F.A.; Resources, B.L.N. and F.A.; Supervision, F.A.; Validation, A.M.-D.1.T., A.C., Q.D. and F.P.B.; Visualization, A.M.-D.1.T., J.T. and F.P.B.; Writingoriginal draft, F.A.; Writing-review \& editing, B.L.N., F.A., M.D., A.C., C.M. and F.G. All authors have read and agreed to the published version of the manuscript.

Funding: This research was supported by an unrestricted grant from Danone Nutricia Research. Danone Nutricia Research authors participated in the study design, interpretation of the data and in the writing of the report. This work was supported in part by the Spanish Ministry of Economy and Competitiveness (Dirección General de Investigación Científica y Técnica, SAF 2016-76648-R). Ciberehd is funded by the Instituto de Salud Carlos III.

Institutional Review Board Statement: The clinical study was conducted according to the Declaration of Helsinki. The study protocol had previously been approved by the Institutional Review Board of the University Hospital Vall d'Hebron (Comitè d'Ètica d'Investigació Clinica, Vall d'Hebron Insititut de Recerca; protocol number PR-AG 292/2012, approved 29 January 2016) and all participants provided written informed consent. The protocol was also registered with ClinicalTrials.gov (NCT02936713).

Informed Consent Statement: Informed consent was obtained from all subjects involved in the study.

Data Availability Statement: Data were uploaded in NCBI with the following accession number: PRJNA789678.

Acknowledgments: The authors want to thank Liliana Galetescu, Marie-Aline Tremolet Rezaï, Bérangère Lefevre and Vanessa Moulinier for project management support; Stéphanie Ploix, Laurence Liang, Stéphanie Cools Portier and Aurore Tessier for analytical support; Sandra Sevaux, Alexandre Grundstein and Lauriane Raidot for data management support; Gloria Santaliestra for secretarial assistance.

Conflicts of Interest: B.L.N., J.T., M.D., J.M.F., A.C. and Q.D. are Danone Nutricia Research employees. F.A. has received unrestricted research grants from Danone Nutricia Research. Other authors have nothing to disclose.

\section{References}

1. Guyonnet, D.; Schlumberger, A.; Mhamdi, L.; Jakob, S.; Chassany, O. Fermented milk containing bifidobacterium lactis dn-173 010 improves gastrointestinal well-being and digestive symptoms in women reporting minor digestive symptoms: A randomised, double-blind, parallel, controlled study. Br. J. Nutr. 2009, 102, 1654-1662. [CrossRef] [PubMed]

2. Guyonnet, D.; Woodcock, A.; Stefani, B.; Trevisan, C.; Hall, C. Fermented milk containing bifidobacterium lactis dn-173 010 improved self-reported digestive comfort amongst a general population of adults. A randomized, open-label, controlled, pilot study. J. Dig. Dis. 2009, 10, 61-70. [CrossRef] [PubMed] 
3. Marteau, P.; Guyonnet, D.; Lafaye de Micheaux, P.; Gelu, S. A randomized, double-blind, controlled study and pooled analysis of two identical trials of fermented milk containing probiotic bifidobacterium lactis cncm i-2494 in healthy women reporting minor digestive symptoms. Neurogastroenterol. Motil. 2013, 25, 331-e252. [CrossRef]

4. Marteau, P.; Le Neve, B.; Quinquis, L.; Pichon, C.; Whorwell, P.J.; Guyonnet, D. Consumption of a fermented milk product containing bifidobacterium lactis cncm i-2494 in women complaining of minor digestive symptoms: Rapid response which is independent of dietary fibre intake or physical activity. Nutrients 2019, 11, 92. [CrossRef] [PubMed]

5. Agrawal, A.; Houghton, L.A.; Morris, J.; Reilly, B.; Guyonnet, D.; Goupil Feuillerat, N.; Schlumberger, A.; Jakob, S.; Whorwell, P.J. Clinical trial: The effects of a fermented milk product containing bifidobacterium lactis dn-173 010 on abdominal distension and gastrointestinal transit in irritable bowel syndrome with constipation. Aliment. Pharmacol. Ther. 2009, 29, 104-114. [CrossRef]

6. Guyonnet, D.; Chassany, O.; Ducrotte, P.; Picard, C.; Mouret, M.; Mercier, C.H.; Matuchansky, C. Effect of a fermented milk containing bifidobacterium animalis dn-173 010 on the health-related quality of life and symptoms in irritable bowel syndrome in adults in primary care: A multicentre, randomized, double-blind, controlled trial. Aliment. Pharmacol. Ther. 2007, 26, 475-486. [CrossRef]

7. Le Nevé, B.; Derrien, M.; Tap, J.; Brazeilles, R.; Cools Portier, S.; Guyonnet, D.; Ohman, L.; Störsrud, S.; Törnblom, H.; Simrén, M. Fasting breath $\mathrm{h} 2$ and gut microbiota metabolic potential are associated with the response to a fermented milk product in irritable bowel syndrome. PLoS ONE 2019, 14, e0214273. [CrossRef]

8. Le Neve, B.; de la Torre, A.M.; Tap, J.; Derrien, M.; Cotillard, A.; Barba, E.; Mego, M.; Nieto Ruiz, A.; Hernandez-Palet, L.; Dornic, Q.; et al. A fermented milk product with b. Lactis cncm i-2494 and lactic acid bacteria improves gastrointestinal comfort in response to a challenge diet rich in fermentable residues in healthy subjects. Nutrients 2020, 12, 320. [CrossRef]

9. Barba, E.; Burri, E.; Accarino, A.; Cisternas, D.; Quiroga, S.; Monclus, E.; Navazo, I.; Malagelada, J.R.; Azpiroz, F. Abdominothoracic mechanisms of functional abdominal distension and correction by biofeedback. Gastroenterology 2015, 148, 732-738. [CrossRef] [PubMed]

10. Burri, E.; Barba, E.; Huaman, J.W.; Cisternas, D.; Accarino, A.; Soldevilla, A.; Malagelada, J.R.; Azpiroz, F. Mechanisms of postprandial abdominal bloating and distension in functional dyspepsia. Gut 2014, 63, 395-400. [CrossRef]

11. Malagelada, C.; Drozdzal, M.; Segui, S.; Mendez, S.; Vitria, J.; Radeva, P.; Santos, J.; Accarino, A.; Malagelada, J.R.; Azpiroz, F. Classification of functional bowel disorders by objective physiological criteria based on endoluminal image analysis. Am. J. Physiol. Gastrointest. Liver Physiol. 2015, 309, G413-G419. [CrossRef] [PubMed]

12. Manichanh, C.; Eck, A.; Varela, E.; Roca, J.; Clemente, J.C.; Gonzalez, A.; Knights, D.; Knight, R.; Estrella, S.; Hernandez, C.; et al. Anal gas evacuation and colonic microbiota in patients with flatulence: Effect of diet. Gut 2014, 63, 401-408. [CrossRef] [PubMed]

13. Mego, M.; Accarino, A.; Tzortzis, G.; Vulevic, J.; Gibson, G.; Guarner, F.; Azpiroz, F. Colonic gas homeostasis: Mechanisms of adaptation following host-g904 galactooligosaccharide use in humans. Neurogastroenterol Mot. 2017, 29, e13080. [CrossRef]

14. Serra, J.; Azpiroz, F.; Malagelada, J.R. Intestinal gas dynamics and tolerance in humans. Gastroenterology 1998, 115, 542-550. [CrossRef]

15. Pascal, V.; Pozuelo, M.; Borruel, N.; Casellas, F.; Campos, D.; Santiago, A.; Martinez, X.; Varela, E.; Sarrabayrouse, G.; Machiels, K.; et al. A microbial signature for crohn's disease. Gut 2017, 66, 813. [CrossRef] [PubMed]

16. Barber, C.; Mego, M.; Sabater, C.; Vallejo, F.; Bendezu, R.A.; Masihy, M.; Guarner, F.; Espin, J.C.; Margolles, A.; Azpiroz, F. Differential effects of western and mediterranean-type diets on gut microbiota: A metagenomics and metabolomics approach. Nutrients 2021, 13, 2638. [CrossRef] [PubMed]

17. Bendezu, R.A.; Mego, M.; Monclus, E.; Merino, X.; Accarino, A.; Malagelada, J.R.; Navazo, I.; Azpiroz, F. Colonic content: Effect of diet, meals, and defecation. Neurogastroenterol. Motil. 2017, 29, e12930. [CrossRef] [PubMed]

18. Agostini, S.; Goubern, M.; Tondereau, V.; Salvador-Cartier, C.; Bezirard, V.; Lévèque, M.; Keränen, H.; Theodorou, V.; Bourdu-Naturel, S.; Goupil-Feuillerat, N.; et al. A marketed fermented dairy product containing bifidobacterium lactis $\mathrm{cncm}$ i-2494 suppresses gut hypersensitivity and colonic barrier disruption induced by acute stress in rats. Neurogastroenterol. Motil. 2012, 24, 376-e172. [CrossRef]

19. Tillisch, K.; Labus, J.; Kilpatrick, L.; Jiang, Z.; Stains, J.; Ebrat, B.; Guyonnet, D.; Legrain-Raspaud, S.; Trotin, B.; Naliboff, B.; et al. Consumption of fermented milk product with probiotic modulates brain activity. Gastroenterology 2013, 144, 1394-1401.e1394. [CrossRef]

20. Wastyk, H.C.; Fragiadakis, G.K.; Perelman, D.; Dahan, D.; Merrill, B.D.; Yu, F.B.; Topf, M.; Gonzalez, C.G.; Van Treuren, W.; Han, S.; et al. Gut-microbiota-targeted diets modulate human immune status. Cell 2021, 184, 4137-4153.e4114. [CrossRef] [PubMed]

21. Huaman, J.W.; Mego, M.; Manichanh, C.; Canellas, N.; Canueto, D.; Segurola, H.; Jansana, M.; Malagelada, C.; Accarino, A.; Vulevic, J.; et al. Effects of prebiotics vs a diet low in fodmaps in patients with functional gut disorder. Gastroenterology 2018, 155, 1004-1007. [CrossRef] [PubMed]

22. Azpiroz, F.; Hernandez, C.; Guyonnet, D.; Accarino, A.; Santos, J.; Malagelada, J.R.; Guarner, F. Effect of a low-flatulogenic diet in patients with flatulence and functional digestive symptoms. Neurogastroenterol. Motil. 2014, 26, 779-785. [CrossRef] [PubMed]

23. Bohn, L.; Storsrud, S.; Liljebo, T.; Collin, L.; Lindfors, P.; Tornblom, H.; Simren, M. Diet low in fodmaps reduces symptoms of irritable bowel syndrome as well as traditional dietary advice: A randomized controlled trial. Gastroenterology 2015, 149, 1399-1407. [CrossRef] [PubMed] 
24. Halmos, E.P.; Power, V.A.; Shepherd, S.J.; Gibson, P.R.; Muir, J.G. A diet low in fodmaps reduces symptoms of irritable bowel syndrome. Gastroenterology 2014, 146, 67-75. [CrossRef]

25. Brokes, S.; Costa, M. Functional neuroanatomy. In Pathophysiology of the Enteric Nervous System; Spiller, R., Grundy, D., Eds.; Blackwell: Oxford, UK, 2004; pp. 126-133.

26. Ford, A.C.; Quigley, E.M.; Lacy, B.E.; Lembo, A.J.; Saito, Y.A.; Schiller, L.R.; Soffer, E.E.; Spiegel, B.M.; Moayyedi, P. Efficacy of prebiotics, probiotics, and synbiotics in irritable bowel syndrome and chronic idiopathic constipation: Systematic review and meta-analysis. Am. J. Gastroenterol. 2014, 109, 1547-1561. [CrossRef] [PubMed] 